\title{
Assessing Reliability of Resiliency Belief Scale (RBS) in the Malaysian Context
}

\author{
A.M. Azlina ${ }^{1}$, Shahrir Jamaluddin ${ }^{2}$ \\ University Malaysia Terengganu ${ }^{1}$, University of Malaya ${ }^{2}$, Malaysia \\ azlina@umt.edu.my,jamaluddinshahrir@um.edu.my
}

\begin{abstract}
Resilient person has the ability to tolerate pain, has self-respect, ability to bounce back, ability to make friends, has freedom to depend on others and person-environment fit [4; 9]. Therefore, resiliency is related to the individual quality or strength that increases his/her ability to withstand adversity [11]. This paper makes an attempt to test empirically the measurement/CFA model of adolescent resiliency using structural equation modeling (SEM). The adolescents' conceptions of resiliency measured such dimensions as active skills, future orientation, risk taking and independence. This study was done based on a total sample of 308 secondary school students from east-coast of peninsular Malaysia. Crossvalidation was done using samples of 308 students. The Resiliency Belief Scales (Mrazek andMrazek) which was adapted from [25], was used in this study. The instrument consisted of 45 items and the items were hypothesized a priori to have non-zero loadings on all dimensions in the model. Analysis of the items resulted in a well-fitting model $\left(\chi^{2}=1.327 ; d f=1\right.$; $G F I=.998 ; C F I=.996 ; T L I=.995 ; p=.35$; Hoelter $=395 ;$ RMSEA $=.03$ ).
\end{abstract}

\section{Introduction}

Human resilience has been described in many different ways. Resilience and vulnerability are often viewed as opposite extremity of continuum reflecting defenselessness to adverse consequences upon exposure to high risk situations [14]. We cannot control events but we can control reactions to the events. Resiliency is the ability to recover readily from illness, depression, adversity, or the like [19]. Resilience also refers to sustained competent functioning despite severely challenging circumstances [6]. Thus, resilience implies effective coping, which including thought and action as the person puts effort to restore or maintain equilibrium under certain threat.

In the resiliency model, resiliency is not a statetrait characteristic, but a response to stimuli. As stress-resistance, resilience is thought to be related to cognitive appraisals of life stressors and the range of problem-solving skills available to individuals. As recovery, resilience is the capacity to recover from traumatic events as the threat recedes in intensity over time [6]. The field of resilience has evolved since the early studies by researchers [21] and [26]. The research focused primarily on identifying intrinsic and extrinsic factors that would either protect or put a child at risk of developing negative outcomes after experiencing difficulties [18]. Richardson et al., as cited in [6], formulated the resiliency model in which they denote competency and resiliency as strengths. They define resiliency as "the process of coping with disruptive, stressful or challenging life events in a way that provides the individual with additional protection and coping skills than prior to the disruption that results from the event".

Continued future studies on resiliency are important because review of previous research literatures found that resiliency correlates positively to academic achievement [22], religiosity [18], selfefficacy [8]; [12], school attachment [18], selfimprovement [16] and well-being [7].

Resiliency should be established among students because it can protect them against health risk behaviors such as suicide ideation or attempts [18]. Emerging research in resiliency has established that predictable and malleable characteristics of certain high risk students appear to support their educational and personal success despite very difficult life circumstances [24]. This study focused on adolescents because as individuals age from childhood to adulthood, they pass through a critical period during which the characteristics of maturity, cognitive skills, coping skills and relationships develop. The myriad changes involve emotional and social stress. According to Resnick, Harrick and Blum as cited in [20], the major health risks facing the estimated 34 million adolescents in the U.S. today are traceable to psychosocial, behavioral and economic factors [18]. In 2006, Malaysian government has approved additional expenses of RM6.1 million per year to handle social problems, especially among adolescents [17].

By focusing on strength or resiliency, we can recognize the potential each individual has. Literature reviews have given more emphasis on risk factors compared to factors that contribute strengths [3]. Furthermore, resiliency can function as innate drives to achieve self-actualization, increased 
motivational energy to grow, potential buffer to stress, capacity to bounce back, recover from adversity and sustainable development among adolescents.

In order to conduct empirically fruitful future research involving resiliency, we need a psychometrically sound measure of resiliency scales. The Resiliency Belief Scale, developed as a general measure of the subjective quality of life, is a widely used 45 -item self-report measure of resiliency. This scale is based on Mrazek and Mrazek, as cited in [25]. Conceptualization of Resiliency Belief Scales is based on three aspects; active skills, future orientation, risk taking and independence.

Individuals who score higher on the resiliency scale are likely to demonstrate better academic skills, have a higher internal locus of control orientation, have higher self-perceived competence in jobs, athletic performance and friendships; and display a wider range of coping skills than are less resilient peers [13].

Further research with the Resiliency scale is needed [13]. Previous studies investigating psychometric features of Resiliency Belief Scale involved English speaking samples. Local data pertaining reliability, validity and factor structure of Resiliency Belief Scale (RBS) are lacking. Therefore, the present study attempted to evaluate the Resiliency Belief Scale involving Malaysian samples. Analysis of measurement model is done by Structural Equation Modeling (SEM). SEM has become increasingly popular in counseling, psychology and rehabilitation research [8]. The main purpose of this study is to evaluate reliability, validity and dimensionality of Resiliency Belief Scale (RBS). Specifically, this study would like to determine whether the RBS is a well-fitted model in Malaysian context.

\section{Sample}

Sample size is an important consideration in SEM analysis, as low sample size has some disadvantages, including low power to detect significant path coefficients and sampling error in the covariance matrix, which can lead to reduction on fit indices [8]. Mueller suggested that the ratio of number of samples to number of observed variables should be at least 10 to 1 [8]. Bollen recommended a ratio of 3 to 5 samples per estimated parameter, whereas Bentler and Chou recommended 5 to 10 samples per estimated parameter [8]. In general, statistical indices will perform sufficiently and yield meaningful and interpretable values when the sample size is 200 or more samples [8].

The total participants in this study were 308 representing $45 \%$ male and $55 \%$ female participants. All of them were 16 years old. The first group of participant involved 160 students was randomly selected from secondary schools from form four in east coast of peninsular Malaysia. Approved letter from Ministry of Education and State Department of Education were attached with the questionnaire. The questionnaire was group administered to the students during their regular class hours with permission from their school principals and teachers. The participants were given brief description of the research project and the process of informed consent was done. To further validate the well-fitted measurement model, a total of 148 students from secondary schools were randomly selected for this study. The same data collection procedures were employed. It took about two months to collect the data.

\section{Instrument}

Resiliency Belief Scale is a self-assessment instrument, based on Mrazek and Mrazek, and developed by Jew, as cited in [25]. They have identified twelve skills and abilities; rapid responsivity to danger, precocious maturity, disassociation of affect, information seeking, formation and utilization of relationships for survival, positive projective anticipation, decisive risk-taking, the conviction of being loved, idealization of aggressor's competence, and cognitive restructuring of painful events [13].

Conceptualization of Resiliency Scales is based on three factors; active skills, future orientation, risk taking and independence. The active skills focus on the skills needed to be resilient such as rapid responsivity to danger in order to avoid harm, information seeking and cognitive restructuring of painful events. Future orientation is related to the conviction of being loved, optimism, altruism, formation of relationship for survival, positive projective anticipation and hope. Risk taking and independence are related to ability of the adolescents to take risk or decisive risk taking, confident, competence and independent.

Each subscale consists of 15 items. Back to back translation was done and was referred to two experts in English and Malay languages. The instrument was based on a cognitive appraisal theory of resiliency first posed by Mrazek and Mrazek. Their theory posited that responses to stress are influenced by appraisal of the situation and the capacity to incorporate the experience into one's belief system. Promotion of resiliency lies in encountering stress at a time and in a way that allows individual to experience appropriate responsibility, thus increasing sense of self-confidence and competence. Resilient persons cope with stress because they use certain skills and abilities to recover from adversity.

The students were required to indicate their beliefs and conceptions of the resiliency assessment of RBS on a six-point response scale (Strongly Agree, Moderately Agree, Agree, Disagree, 
Moderately Disagree and Strongly Disagree). Each statement was worded in a manner to capture the meaning attached to one of the three dimensions.

\section{Data Analysis}

Analysis of data involves the measurement by Structural Equation Modeling (SEM). In SEM, a construct can be tested as both a predictor construct and a criterion construct [8]. Since a construct is assumed to be measured with error, SEM procedures can be used to calculate the reliability of both measurement instruments and estimated latent construct. Furthermore, SEM enables evaluation of the general compatibility of the model, that is goodness of fit, as well as the strength of the relationships among constructs [8].

The main task in this model testing procedure is to determine the goodness of fit between the hypothesized model and the sample data. To arrive at the conclusion, a confirmatory factor analysis (CFA) was conducted on the hypothesized three-factor structure model using Analysis of Moment Structure (AMOS) version 7. Assessment of the measurement model involved confirmatory factor analysis (CFA) [5]. CFA assessed the reliability and validity of the individual items and the overall measurement model. The program adopted maximum likelihood estimation to generate estimates in the measurement model.

To assess for the reliability of the instrument in this study the researcher makes use of estimates of internal consistency (Cronbach's alpha). Cronbach'a alpha is a commonly used measure testing the extent to which multiple indicators for a latent variable belong together. A general rule is that the indicators should have a Cronbach's alpha of 0.70 or more [23].

Further confirmation of the overall fit of the measurement model using CFA is obtained from the Maximum Likelihood estimation Chi-Square $\left(\chi^{2}\right)$ statistics produced by AMOS and various other goodness-of-fit criteria. Chi-square is the most common method of evaluating goodness-of-fit.

Byrne suggested the goodness of fit indexes are the chi-square $\left(\chi^{2}\right)$, the smaller the chi square, the better and $p$ value greater than .05, Goodness of Fit Index (GFI) and Comparative Fit Index (CFI) are greater than .90 and the absolute fit of the model, Root Mean Square Error of Approximation (RMSEA) is below .08. Marten (2005) indicated that chi-square/df, GFI and NFI (normed fit index) tend to be substantially affected by sample size [8]. Marten suggested using CFI and RMSEA as the primary goodness of fit indexes. The purpose of Hoelter is to estimate a sample size that would be sufficient to yield an adequate model fit for a chisquare test [5]. AMOS reports a 90\% confidence interval around the RMSEA value [8]. Subsequently the final fitted measurement model was further validated.

\section{Results}

The validity and reliability of the instrument reported here are based on the data collected from this study. The strategy employed in the present study to test the validity and the reliability of the instrument focuses on the psychometric properties of the scales used. In this aspect the researcher makes use of estimates of internal consistency (Cronbach's alpha) and confirmatory factor analysis (CFA).

Table 1 shows the internal consistency (Cronbach's alpha) above the satisfactory value of .70 [1].Table 2 shows the regression weights of the model and Table 3 shows the goodness of fit indexes of confirmatory factor analysis (CFA) for the measurement model of adolescents resiliency by using Resiliency Belief Scale.

Table 1. Internal Consistency of RBS (Cronbach's alpha)

\begin{tabular}{|l|c|}
\hline \multicolumn{1}{|c|}{ Subscales in RBS } & Reliability \\
\hline Future orientation & .93 \\
\hline Active skills & .91 \\
\hline $\begin{array}{l}\text { Risk taking and } \\
\text { independence }\end{array}$ & .93 \\
\hline Total instrument & .93 \\
\hline
\end{tabular}

Table 2. Standardized paths of a hypothesized model

\begin{tabular}{|c|l|l|l|c|c|}
\hline Hypotheses & & & \multicolumn{1}{|c|}{ Causal Path } & C.R. & $\begin{array}{c}\text { Standardized Path } \\
\text { Coefficient }\end{array}$ \\
\hline H1 & par1 & $<---$ & Future Orientation & - & - \\
\hline H2 & par2 & $<---$ & Future Orientation & 5.664 & $* * *$ \\
\hline H3 & par3 & $<---$ & Future Orientation & 4.278 & $* * *$ \\
\hline H4 & par4 & $<---$ & Future Orientation & 5.864 & $* * *$ \\
\hline H5 & par5 & $<---$ & Future Orientation & 5.957 & $* * *$ \\
\hline H6 & par6 & $<---$ & Active skill & - & - \\
\hline H7 & par7 & $<---$ & Active skill & 9.190 & $* * *$ \\
\hline H8 & par8 & $<---$ & Active skill & 6.111 & $* * *$ \\
\hline H9 & par9 & $<---$ & Active skill & 6.890 & $* * *$ \\
\hline H10 & par10 & $<---$ & Active skill & 10.119 & $* * *$ \\
\hline H11 & par11 & $<---$ & Risk Taking & 9.835 & $* * *$ \\
\hline H12 & par12 & $<---$ & Risk Taking & 11.022 & $* * *$ \\
\hline H13 & par13 & $<---$ & Risk Taking & 11.811 & $* * *$ \\
\hline H14 & par14 & $<---$ & Risk Taking & 10.412 & $* * *$ \\
\hline H15 & par15 & $<---$ & Risk Taking & - & - \\
\hline
\end{tabular}

Note: $* * *=\mathrm{P}<.0001$

Table 3. Measures of Goodness of Fit for the Measurement Model

\begin{tabular}{|c|c|c|c|}
\hline Latent Variables (RBS) & N1 $=160$ & $\mathrm{~N} 2=148$ & $\mathrm{~N} 3=308$ \\
\hline$d f$ & 4 & 4 & 1 \\
\hline$\chi^{2}$ & 7.22 & 7.38 & 1.33 \\
\hline$p$ & .38 & .21 & .35 \\
\hline TLI & .999 & .995 & .995 \\
\hline GFI & .94 & .95 & .998 \\
\hline CFI & .94 & .92 & .996 \\
\hline RMSEA & .06 & .06 & .03 \\
\hline Hoelter & 211 & 232 & 395 \\
\hline
\end{tabular}




\section{Hypothesized Model and Discussions}

Figure I present the estimated three-factor model for adolescents' resiliency of RBS, using the data from 308 students. Items from each scale are assumed to load only on their respective latent variables. The overall fit of the 45 -item measurement model is summarized in Figure 1. The goodness-offit results indicate the hypothesized model is consistent with the data.

In SEM, a non-significant chi-square value is an indication that the hypothesized model has a good fit with the data [8]. A low chi-square value, indicating non-significance, would point to a good fit [10]. Therefore, non-significance means there is no difference between the actual and predicted matrices. A high ' $p$ ' value is good as it indicates that the observed model is not significantly different from what was expected [10]. However, chi-square is hypersensitive to sample size.

Root Mean Square Error of Approximation (RMSEA) has been recognized as one of the most informative criteria in covariance structure modeling. The RMSEA takes into account the error of approximation in the population [5]. Values less than .05 indicate good fit and values as high as .08 represent reasonable errors of approximation in the population.

For CFI (Comparative Fit Index) and GFI (Goodness of Fit Index), values close to 1.00 being indicative of good fit. Although a value of $>.90$ was originally considered representative of a well-fitting model, a revised cutoff value close to .95 has recently been accepted [5]. Because the CFA model focuses on the link between factors and their measured variables, within the framework of Structural Equation Modeling (SEM), it represents what has been termed a measurement model. The Tucker-Lewis index (TLI) yields values ranging from zero to 1.00 , with values close to .95 being indicative of good fit [5]. Hoelter proposed that a value in excess of 200 is indicative of a model that adequately represents the sample data [5].

All the 45 items have non-zero loadings to the three factors. Refer Table 2 for the maximum likelihood estimation. All the items loaded significantly to the three factors. The direction and magnitude of the factor loadings were substantial and statistically significant. The model is free from offending estimates and the internal consistency estimates satisfied the standard deemed necessary in scale construction. For example, the probability of getting a critical ratio for hypothesis $(\mathrm{H} 1)$, as large as 5.664 in absolute value is less than .0001 . In other words, the regression weight for future orientation in the prediction of par2 is significantly different from zero at the .0001 level (two-tailed). The same explanations are applied to the other hypotheses.
The result of this analysis is presented in Table 3 . Analysis of the 45 items resulted in a well-fitting model for the first analysis, $\mathrm{n}=160\left(\chi^{2}=7.22 ; d f=\right.$ 4 ; $\mathrm{GFI}=.94$; $\mathrm{CFI}=.93$; $\mathrm{RMSEA}=.06)$. Both the fit indicators, the GFI and CFI exceeded the threshold of .90 , the standard deemed important for model fit [5]. Furthermore, the root mean square error of approximation (RMSEA $=.06$ ) indicated a well fitted hypothesized model.

To further validate the likelihood of the hypothesized model, a second confirmatory factor analysis (cross-validation) was applied on the data collected from 148 students. The results of this analysis is presented in Table 3 in cross validation model shows the well-fitting model $\left(\chi^{2}=7.38 ; d f=\right.$ 4 ; $\mathrm{GFI}=.95 ; \mathrm{CFI}=.92 ; \mathrm{RMSEA}=.06)$.

The third validation was done by combining all data, $\mathrm{N}=308$ to further validate the confirmatory factor analysis. The results of the analysis is presented in Table 3 shows that analysis of the items resulted in a well-fitting model $\left(\chi^{2}=1.32 ; d f=1\right.$; $\mathrm{GFI}=.99 ; \mathrm{CFI}=.99$; RMSEA $=.03)$. Therefore, the above results show that RBS is internally consistent measure of resiliency (above the satisfactory value), a valid measure of resiliency, all items loaded significantly to the three factors and it is a well-fitted model of adolescents in Malaysian context.

With regard to dimensionality of the RBS, the current findings indicate that the scale is multidimensional and first-order factor. All items loaded significantly to the three factors (future orientation, active skills, risk taking and independence).

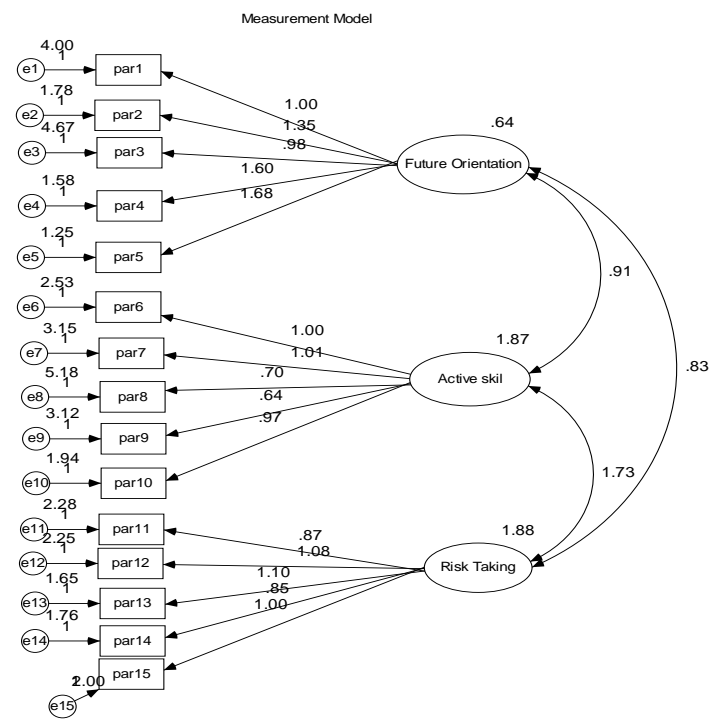

Figure 1. Correlated First-Order Factor
of RBS scale

(par1-par15 represent observed variables; e1-e15 represent error variances; double-headed arrows 
depict correlations among factors; single headed arrows from factors depict factor loadings)

\section{Conclusion}

One major advantage of SEM applied to CFA is that the validity of the a priori expected factor structure can be evaluated on multiple goodness of fit indices [8]. Present findings show that the RBS is a reliable and valid measure of resiliency among adolescents in Malaysia. The study contributes to the development of a psychometrically sound instrument to assess adolescents' resiliency of Resiliency Belief Scale. The results of the confirmatory factor analysis support the assertion that the conception is a multidimensional construct. These findings are consistent with previous studies of resiliency of assessment [1], [2], [25]. Previous studies using this instrument were conducted in English. Evidently, the current study which made use of the same instrument in Malay language yields consistent findings.

This study has implications for educational practices, especially assessing resiliency of adolescents. Since the 45 -item three-factor structure yields a valid and reliable measurement model, the conception scale is therefore, useful in conducting diagnostic assessment of resiliency among adolescents in Malaysia. Future research should examine whether the present findings generalize to other samples and settings. Furthermore, it is important to use the local version of RBS in the future research because many people feel more comfortable in expressing their feelings in their own language as compared to a foreign language. Besides, continued future studies on resiliency are important because resiliency has positive consequences.

\section{References}

[1] Anderson, E., and Shilkret, R. (2004). A New Measure and Conception of Resilience for College Students. http://www.controlmastery.org/docs/Anderson_Shilkret20 04.pdf

[2] Bogdonoff, D. A., (2003). Dissertation Abstracts International :Section B:The Sciences and Engineering. Vol 63(9-B), Mar, 2003. pp. 4361.

[3] Borman, G.D., and Rachuba, L.T. (2001). Academic Success among Poor and Minority Students: An Analysis of Competing Models of School Effects. (Proquest Education Journals Service No. AAT3014236).

[4] Brown, J.H., Caston, M. \& Benard, B. (2001). Resilience Education. Thousand Oaks: Corwin Press, Inc

[5] Byrne, B.M. (2001). Structural Equation Modeling with AMOS: Basic Concepts, Applications and Programming. New Jersey: Lawrence Erlbaum Associates, Pub.
[6] Cabness, J.L. (2003). Psychosocial Resilience, Depression and Subjective Well-Being in Long Term Care. (Proquest Education Journals Service No.AAT3075227)

[7] Carlton, P. (1991). The Experience of Black Male Administrators at White four-year High Institution. (Proquest Education Journals Service No. AAT9132895)

[8] Chan, F., Lee, G.K., Lee, E., Kubota, C., and Allen, C.A. (2007). Structural equation modeling in rehabilitation counseling research. Rehabilitation Counseling Bulletin, 51:1, pp.53-66.

[9] Flach, F., (1988). Resilience: Discovering a New Strength at Times of Stress. New York: Fawcett Columbine.

[10] Hair, J.F., Jr.Anderson, R.E. Tatham, R.L. and Black, W.C. (1998). Mutivariate Data Analysis, New Jersey, Prentice Hall.

[11] Holmes, K., (2006). Adolescent Resilience: The Influence of Family Relationships and their Impact on Resilient Outcomes. (Proquest Education Journals Service No.AAT3223401)

[12] Jenkins, D.K. (2000). A Retrospective Study of Academic and Social Resiliency that can Contribute to Success of At-risk Students. (Proquest Education Journals Service No. AAT 9992220)

[13] Jew, C.L., Grren, K. E., and Kroegr, J., (1999). Development and validation of a measure of resilience. Measurement and Evaluation in Counseling and Development, July, 32, 2, Proquest Education Journals, p.75.

[14] Kaplan, H. B., (1999). Toward an Understanding of Resilience: A Critical Review of Definitions and Models. Now York: Kluwer Academic/Plenum Publishers.

[15] Kaplan, D. (2000). Structural Equation Modeling: Foundations and Extensions. Advanced Quantitative Techniques in the Social Sciences Series 10. Thousand Oaks: Sage.

[16] Langer, N., (2004). Resiliency and Spirituality: Foundations of Strengths Perspective Counseling with the Elderly. Educational Gerontology, 30, 611-617

[17] Malaysia's Education Ministry, (2006). Formulation of National Education Blueprint 2006-2010. Kuala Lumpur: Bahagian Perancangan dan Penyelidikan Dasar Pendidikan.

[18] Morrison, C. S., (2003). Connectedness and Religiosity As Protective Factors: Enhancing Health Promotion among Parochial High School Adolescents. (Proquest Education Journals Service No.AAT1413067).

[19] Rak, C.F. and Lesiw, E. (1996). Promoting Resilience in At-risk Children, Journal of Counseling and Development, vol. 74., 4, p.368. 
[20] Resnick, Harrick and Blum, (1993). In Morrison, C.S. (2003). Connectedness and Religiosity As Protective Factors: Enhancing Health Promotion among Parochial High School Adolescents. (Proquest Education Journals Service No.AAT1413067).

[21] Rutter, (1985). In Flach, F., (1988). Resilience: Discovering a New Strength at Times of Stress. New York: Fawcett Columbine.

[22] Shaughnessy, E., S. M. Suldo, et al., (2006). School Functioning and Psychological Well-Being of International Baccalaureate and General Education Students. The Journal of Secondary Gifted Education 17(2), 76.

[23] Spector, P. E., (1992). Summated rating Scale Construction: An Introduction. Newbury Park, CA: SAGE Publication.

[24] Thomson, C.W., (2005). Fostering Coping Skills and Resilience in Home Enteral NutritionConsumers. Nutrition in Clinical Practice:Official Publication of American Society for Parenteral and Enteral Nutrition. Dec; Vol. 21 (6), pp. 557-65.

[25] Trammel, M.S., (2003). The Effects of Caring Adults, Religiosity and Resiliency on African American Middle School Girls' School Attachment, Self-Concept and Participation in Out-of-School Time Programs. (Proquest Education Journals Service No.AAT3114650).

[26] Werner, E.E. and Smith, R.S. (1992). Vulnerable but Invisible: A Study of Resilient Children. New York: McGraw Hill. 